\title{
Efecto del tratamiento ortodóncico en los tejidos periodontales: revisión de literatura
}

Ceccarelli-Calle JF, Alania-Mallqui JA, Alarcón-Palacios MA. Efecto del tratamiento ortodóncico en los tejidos periodontales: revisión de literatura. Rev Estomatol Herediana. 2010; 20(4):216220 .

RESUMEN

El tratamiento ortodóncico puede producir una estética dentofacial muy agradable, una oclusión funcional superior. Sin embargo, a lo largo de los años se han reportado resultados contradictorios respecto al efecto del tratamiento ortodóncico sobre los tejidos periodontales. Esta revisión de literatura recolecta la información disponible sobre los efectos clínicos, histológicos, radiológicos y microbiológicos del tratamiento ortodóncico.

Palabras clave: ORTODONCIA / PERIODONCIA / APARATOLOGÍA ORTODÓNCICA / HISTOLOGÍA / RADIOLOGÍA / MICROBIOLOGÍA.

Effect of the orthodontic treatment in the periodontal tissues: a review ABSTRACT

Orthodontic treatment can produce a very nice dentofacial aesthetic, a superior functional occlusion. However, over the years it had been reported conflicting results regarding the effect of orthodontic treatment on periodontal tissues. This literature review collects the available information about the clinical, histological, radiological and microbiological aspect of the orthodontic treatment.

Keywords: ORTHODONTICS / PERIODONTICS / ORTHODONTIC APPLIANCES / HISTOLOGY / RADIOLOGY / MICROBIOLOGY.

\section{Introducción}

El tratamiento de ortodoncia puede producir una estética dentofacial muy agradable, una oclusión funcional superior (1).

La ortodoncia constituye una disciplina de la estomatología que diagnostica y da tratamiento a la maloclusión. Ésta se define como una alteración del crecimiento y desarrollo que compromete la oclusión dentaria, siendo considerada como un problema de salud pública por su alta prevalencia, ya que afecta de manera negativa en la calidad de vida, perjudicando la interacción social y el buen desenvolvimiento psicológico del individuo afectado (2).

Los aparatos ortodónticos son aquellos dispositivos empleados durante el tratamiento de la maloclusión. Entre estos distinguimos a los aparatos fijos y removibles de acuerdo a la capacidad de ser retirados de la boca por el propio paciente. Su función se realiza mediante la generación de fuerzas que conllevan al desplazamiento dentario (3). Su presencia contribuye al incremento de áreas retentivas de acumulo de placa bacteriana (4). El objetivo de esta revisión de literatura es realizar una recopilación de la información actual existente sobre el efecto del tratamiento ortodóncico en los tejidos periodontales.

\section{Evidencia clínica}

En denticiones apiñadas donde la mal posición constituye un factor de riesgo para el desarrollo de enfermedad gingival, se ha demostrado que el tratamiento ortodóncico produce un efecto favorable a largo plazo $(5,6)$.

Diversos estudios han evaluado el efecto clínico del tratamiento ortodóncico sobre los tejidos gingivales. Se ha encontrado que la aparatología se relaciona al incremento significativo de sangrado a la exploración y de índice de placa bacteriana (7), no observándose un incremento de profundidad al sondeo (7-9). Sin embargo, otros estudios han reportado un leve incremento de la profundidad al sondeo en las superficies de las piezas con aparatología, esto asociado al movi-
Juan Francisco Ceccarelli Calle ${ }^{1}$ Jorge Alania Mallqui ${ }^{1}$ Marco Alarcón Palacios ${ }^{2}$

'Residente del Programa de Especialización en Periodoncia e Implantes dentales.

2Docente del Departamento Académico de Clinica Estomatológica.

Facultad de Estomatología. Universidad Peruana

Cayetano Heredia.

\section{Correspondencia}

Juan Francisco Ceccarelli Calle

Jr. Pedro Paulet 316 -401 - Lima 31; Perú

Teléfono: 945119290.

e-mail: juan.ceccarelli.c@upch.pe

uan_ceccarelli@ hotmail.com

Recibido : 18 de octubre de 2010

Aceptado : 16 de noviembre de 2010 miento y posición dentaria $(10,11)$. Zachrisson (12) reportó que la profundidad al sondeo se incrementa entre 0,2 a $0,37 \mathrm{~mm}$, encontrando una pérdida de nivel de inserción clínico de 0,41+0,52 mm en el grupo experimental, siendo de $0,11+0,16 \mathrm{~mm}$ en el grupo control que no recibió aparatología. Se ha observado una mayor recesión de tejidos marginales (13) siendo mayor su incidencia en las piezas antero inferiores (14), donde la inclinación y grosor de los tejidos gingivales constituyen factores de riesgo para el desarrollo de las recesiones (15). Bollen (16) en 2008 realizó una revisión de literatura encontrando que a la aparatología ortodóncica se asocia una recesión de tejidos marginales de $0,03 \mathrm{~mm}$, concluyendo que el efecto clínico de la presencia de la aparatología ortodóntica es mínimo.

Respecto a la evolución negativa de los parámetros clínicos, Ristic (17) reportó que los máximos valores son obtenidos después de tres meses de la instalación de la aparatología. Kouraki (18) reportó que el agrandamiento gingival es una 
condición común durante el tratamiento ortodóntico con aparatología fija, siendo su prevalencia de 53,3\%. Así mismo reportó que después de la remoción de la aparatología no se observa una resolución completa de esta alteración. Kloehn (19) reportó que la presencia de agrandamiento gingival fue cuatro veces más frecuente en piezas posteriores y zonas interproximales que en piezas anteriores y superficies vestibulares respectivamente, siendo las piezas molares superiores más afectadas respecto a las molares inferiores. Así mismo, el acúmulo de placa bacteriana que conlleva a la inflamación se ha observado con mayor frecuencia en las superficies proximales y en pacientes adolescentes frente a adultos (20). Se ha demostrado que en ausencia de placa no se produce inflamación en pacientes con aparatología, lo cual refuerza el concepto de la presencia de placa bacteriana como factor etiológico de la enfermedad (21). Sin embargo, otros autores han observado resultados contradictorios, reportando al agrandamiento gingival como una secuela común del tratamiento ortodóncico, incluso en pacientes con buena higiene oral (22). Burch (23) evaluó el efecto del tratamiento ortodóncico sobre el área de furcación en pacientes con enfermedad periodontal previa en 20 molares (40 áreas con compromiso de furcación) en 16 pacientes, evaluándose los hallazgos a los 28 meses. Se encontró que 9 de 20 furcaciones vestibulares habían incrementado su compromiso, 1 mostró mejora y 10 permanecieron sin cambios. Respecto a la cara lingual, 9 incrementaron su compromiso y 11 no presentaron cambios. La profundidad de la bolsa del área de furcación se incrementó en el $35 \%$ de las furcaciones, sin embar- go no se observaron cambios en un $57,5 \%$ de estas. Uno de los factores que contribuyó a los cambios lo constituyó una posible extrusión de la raíz mesial en un $60,0 \%$ de las molares verticalizadas.

\section{Evidencia histológica}

Zachrisson (4) evaluó muestras de tejido gingival obtenido de individuos de 11 a 15 años durante su tratamiento ortodóntico. Las biopsias obtenidas al inicio del tratamiento contenían invariablemente algunas células inflamatorias, mayormente linfocitos, los cuales se encontraban dispersos a lo largo de epitelio del surco. Las biopsias obtenidas en las últimas citas del tratamiento presentaron un incremento de infiltrado de células mononucleares, células plasmáticas y linfocitos. Durante los primeros meses lo cambios fueron subcrónicos observándose una predominancia de linfocitos y tan solo un pequeño número de células plasmáticas en las regiones centrales. Estos cambios persistieron durante los primeros meses incluso en pacientes con buena higiene oral. Sin embargo, en la mayoría de sujetos el cuadro evolucionó a un infiltrado inflamatorio crónico. Respecto a la ubicación del componente celular, se observó que las células plasmáticas se localizaban principalmente a lo largo del tejido conectivo con predominancia en los sectores centrales, mientras que los linfocitos eran más comunes adyacentes el epitelio de la bolsa. Un número significativo de neutrófilos se encontraron cercanos el epitelio de la bolsa. Después de la remoción de la aparatología ortodóncica, las alteraciones celulares y vasculares subsistieron varios meses después. Kloehn (19) reportó que el número de osteoclastos en la zona de presión 8 veces mayor que el visto en las zonas control.

\section{Evidencia radiológica}

Ericsson (21) evaluó el efecto del tratamiento ortodóncico sobre el nivel óseo, encontrando que no se producía reabsorción en los individuos con buen control de placa. En los individuos con mal control de placa se observó un incremento del ancho del ligamento periodontal y pequeños defectos óseos.

Zachrisson (24) después de dos años de seguimiento reportó que la distancia entre la unión cemento adamantina y la cresta alveolar era de 1,11 mm en el grupo experimental, siendo de $0,88 \mathrm{~mm}$ en el grupo control que no recibió aparatología.

Bondemark (25) evaluó el nivel óseo proximal después de 2,7 años de tratamiento ortodóncico. En el grupo experimental se observó un incremento de $0,2 \mathrm{~mm}$ de la distancia entre la unión cemento adamantina y la cresta alveolar. En el grupo control se observó un incremento de 0,1mm. Los sitios mesiales de las primeras molares maxilares fueron los más afectados, mostrándolos mayores niveles de pérdida ósea de hasta $0,5 \mathrm{~mm}$. Ninguno de los sitios presentó una pérdida ósea que superase los $2 \mathrm{~mm}$. Así mismo, Bollen (16) en 2008 realizó una revisión de literatura encontrando que la aparatología ortodóncica producía una pérdida de nivel óseo de $0,23 \mathrm{~mm}$, concluyendo que el efecto clínico de la presencia de la aparatología ortodóncica es mínimo.

\section{Evidencia microbiana}

Demling (26) en 2009 demostró discrepancias respecto a la formación del biofilm supra e infra gingival, encontrando una diferencia significativa y marcada entre ambos. La presencia de biofilm supragingival no necesariamente compromete la pre- 
sencia de biofilm infragingival maduro, sin embargo, Van Gastel (11) encontró que el ratio entre cantidad de bacterias supra e infragingivales disminuye significativamente durante el tratamiento ortodóncico. Thornberg (27) evaluó los niveles de patógenos periodontales en adolescentes, antes, durante y después del tratamiento ortodóncico con aparatología fija, encontrando que los patógenos Prevotella intermedia, Tannerella forsythia y Fusobacterium nucleatun se incrementan significativamente en número después de seis meses de la instalación de la aparatología. Sin embargo, otros autores han reportado que su número vuelve a los niveles de pretratamiento después de 3 a 12 meses $(27,28)$. También se ha encontrado la presencia de A $c$ t $i$ n $\quad o$ b a c c i l l l u s actinomycetemcomitans en sujetos con aparatología $(29,30)$. Así mismo, se ha observado un incremento del número de las bacterias como Enterobacter cloacae, Klebsiella oxytoca, Klebsiella pneumoniae y Serratia marcescens (7).

Sallum (29) reportó que la alta carga bacteriana puede volver a los valores normales después de la remoción de la aparatología y el desbridamiento.

\section{Tratamiento ortodóncico en el paciente con pérdida ósea}

Es bien sabido que la combinación de inflamación, fuerzas ortodóncicas y trauma oclusal pueden producir una destrucción más rápida que la observada con tan solo un proceso inflamatorio (31). Existen pocos estudios que evalúen el tratamiento ortodóncico en pacientes con pérdida ósea. Boyd (32) estudió un grupo de adultos con enfermedad periodontal severa, los cuales recibieron tratamiento de la en- fermedad así como una terapia periodontal de soporte cada tres meses y un tratamiento ortodóncico de dos años. Los resultados fueron comparados con los obtenidos en 10 adultos sin pérdida ósea y 20 pacientes en tratamiento ortodóncico. Se concluyó que los adultos son más efectivos que los adolescentes en el control de placa. El movimiento dentario en adultos con periodonto reducido pero sano, no resulta en una pérdida futura de nivel de inserción. Artun y Urbye (33) evaluaron 24 pacientes con pérdida ósea severa a quienes se les sometió a tratamiento ortodóncico por siete meses. La gran mayoría de sitios no presentaron pérdida ósea. Nelson (34) y Re (35), investigando de manera independiente, reportaron que el tratamiento periodontal previo a la terapia ortodóncica no está contraindicado, la terapia ortodóncica mejora las posibilidades de preservación de la dentición y que el riesgo de la recidiva de la enfermedad no se encuentra incrementado por el uso de la aparatología. Sin embargo, se ha podido observar que los adultos son más susceptibles a la pérdida de las estructuras de soporte que los adolescentes. Nelson (34) reportó una pérdida de $0,54 \mathrm{~mm}$, donde solo el 2,5\% de los pacientes presentaban una pérdida ósea promedio mayor o igual a $2 \mathrm{~mm}$, un $36 \%$ de estos pacientes presentaron 1 ó más superficies con pérdida ósea igual o mayor de $2 \mathrm{~mm}$.

\section{Conclusiones}

- El tratamiento ortodóncico no causa un impacto significativo sobre las estructuras periodontales.

- El efecto clínico del tratamiento ortodóncico sobre los tejidos periodontales puede estar constituido por un incremento del sangrado y profundidad al sondeo, de acúmulo de placa bacteriana, recesión de tejidos marginales y agrandamiento gingival.

- El efecto histológico del tratamiento ortodóncico sobre los tejidos periodontales puede estar constituido en un primer momento por un incremento del número de linfocitos y células plasmáticas, sin embargo, a largo plazo se observa

\begin{tabular}{|l|l|}
\hline Efecto del tratamiento ortodóncico en los tejidos periodontales \\
\hline \multirow{4}{*}{ Clínico } & Incremento de sangrado al sondeo. \\
\cline { 2 - 3 } & Incremento índice de placa bacteriana. \\
\cline { 2 - 3 } & Incremento de profundidad al sondeo. \\
\cline { 2 - 3 } & Riesgo de recesión de tejidos marginales. \\
\cline { 2 - 2 } & Agrandamiento gi ngival. \\
\hline Radiológico & $\begin{array}{l}\text { Primeros meses: predominanci a de linfocitos y tan } \\
\text { solo un pequeño número de células plasmáticas. } \\
\text { A largo plazo: infiltrado crónico, células } \\
\text { mononucleares, células plasmáticas y linfocitos. }\end{array}$ \\
\cline { 2 - 2 } Microbiológico & $\begin{array}{l}\text { Incremento de número de osteoclastos en la zona } \\
\text { de presión 8 veces mayor a sitios control. }\end{array}$ \\
\hline & $\begin{array}{l}\text { Incrementida promedio de nivel óseo de 0,2mm. Prevotella intermedia, Tannerella } \\
\text { forsythia y Fusobacterium nucleatum, } \\
\text { Actinobacillus actinomycetemcomitans. Así mismo } \\
\text { de Enterobacter cloacae, Klebsiella oxytoca, } \\
\text { Klebsiella pneumoniae y Serratia marcescens. }\end{array}$ \\
\hline
\end{tabular}

Cuadro propuesto sobre el efecto del tratamiento ortodóncico en los tejidos periodontales. 
un infiltrado crónico de células mononucleares, plasmáticas y linfocitos.

- El efecto radiológico del tratamiento ortodóncico sobre los tejidos periodontales puede estar constituido por una pérdida promedio de nivel óseo de $0,2 \mathrm{~mm}$.

- El efecto microbiológico del tratamiento ortodóncico sobre los tejidos periodontales puede estar constituido por un incremento de los niveles de $P$. intermedia, $T$. forsythia, $F$. nucleatum, A. actinomycetemcomitans.

- El tratamiento ortodóncico no está contraindicado en el paciente con pérdida de soporte severo, sin embargo, el paciente debe de haber respondido de manera favorable al tratamiento y encontrarse en fase de mantenimiento periodontal.

\section{Referencias bibliográficas}

1. Zachrisson BU. Cause and prevention of injuries to teeth and supporting structures during orthodontic treatment. Am J Orthod. 1976; 69(3):285-300.

2. Marques LS, Barbosa CC, Ramos-Jorge ML, Pordeus IA, Paiva SM. [Malocclusion prevalence and orthodontic treatment need in 10-14-year-old schoolchildren in Belo Horizonte, Minas Gerais State, Brazil: a psychosocial focus] [Article in Portuguese]. Cad Saude Publica. 2005; 21(4):1099-106.

3. Canut J. Ortodoncia clínica. Barcelona: Ed. Salvat; 1991.

4. Zachrisson BU. Gingival condition associated with orthodontic treatment. II. Histologic findings. Angle Orthod. 1972; 42(4):353-7.

5. Glans R, Larsson E, Øgaard B. Longitudinal changes in gingival condition in crowded and noncrowded dentitions subjected to fixed orthodontic treatment. Am J Orthod Dentofacial Orthop. 2003; 124(6):679-82.

6. Davies TM, Shaw WC, Worthington HV, Addy M, Dummer P, Kingdon A. The effect of orthodontic treatment on plaque and gingivitis. Am J Orthod Dentofacial Orthop. 1991; 99(2):155-61.

7. Naranjo AA, Triviño ML, Jaramillo A, Betancourth M, Botero JE. Changes in the subgingival microbiota and periodontal parameters before and 3 months after bracket placement. Am J Orthod Dentofacial Orthop. 2006; 130(3):275.e17-22.

8. Demling A, Demling C, Schwestka-Polly R, Stiesch M, Heuer W. Short-term influence of lingual orthodontic therapy on microbial parameters and periodontal status. A preliminary study. Angle Orthod. 2010; 80(3):480-4.

9. Demling A, Demling C, Schwestka-Polly R, Stiesch M, Heuer W. Influence of lingual orthodontic therapy on microbial parameters and periodontal status in adults. Eur J Orthod. 2009; 31(6):638-42.

10. Sinclair PM, Berry CW, Bennett $\mathrm{CL}$, Israelson $\mathrm{H}$. Changes in gingiva and gingival flora with bonding and banding. Angle Orthod. 1987; 57(4):271-8.

11.van Gastel J, Quirynen M, Teughels W, Coucke W, Carels C. Longitudinal changes in microbiology and clinical periodontal variables after placement of fixed orthodontic appliances. J Periodontol. 2008; 79(11):2078-86.

12.Zachrisson BU, Alnaes L. Periodontal condition in orthodontically treated and untreated individuals. I. Loss of attachment, gingival pocket depth and clinical crown height. Angle Orthod. 1973; 43(4):402-11.

13.Levin L, Samorodnitzky-Naveh GR, Machtei EE. The association of orthodontic treatment and fixed retainers with gingival health. J Periodontol. 2008; 79(11):2087-92.

14. Pearson LE. Gingival height of lower central incisors, orthodontically treated and untreated. Angle Orthod. 1968; 38(4):337-9.

15. Yared KF, Zenobio EG, Pacheco W. Periodontal status of mandibular central incisors after orthodontic proclination in adults. Am J Orthod Dentofacial Orthop. 2006; 130(1):6.e1-8.

16.Bollen AM, Cunha-Cruz J, Bakko DW, Huang GJ, Hujoel PP. The effects of orthodontic therapy on periodontal health: a systematic review of controlled evidence. J Am Dent Assoc. 2008; 139(4):413-22.

17. Ristic M, Vlahovic Svabic M, Sasic M, Zelic O. Clinical and microbiological effects of fixed orthodontic appliances on periodontal tissues in adolescents. Orthod Craniofac Res. 2007; 10(4):187-95.

18. Kouraki E, Bissada NF, Palomo JM, Ficara AJ. Gingival enlargement and resolution during and after orthodontic treatment. N Y State Dent J. 2005; 71(4):34-7.

19. Kloehn JS, Pfeifer JS. The effect of orthodontic treatment on the periodontium. Angle Orthod. 1974; 44(2):127-34.

20.Boyd RL, Baumrind S. Periodontal considerations in the use of bonds or bands on molars in adolescents and adults. Angle Orthod. 1992; 62(2):117-26. 
21. Ericsson I, Thilander B, Lindhe J. Periodontal conditions after orthodontic tooth movements in the dog. Angle Orthod. 1978; 48(3):210-8.

22.Zachrisson BU, Zachrisson S. Gingival condition associated with partial orthodontic treatment. Acta Odontol Scand. 1972;30(1):127-36.

23. Burch JG, Bagci B, Sabulski D, Landrum C. Periodontal changes in furcations resulting from orthodontic uprighting of mandibular molars. Quintessence Int. 1992; 23(7):509-13.

24.Zachrisson BU, Alnaes L. Periodontal condition in orthodontically treated and untreated individuals. II. Alveolar bone loss: radiographic findings. Angle Orthod. 1974; 44(1):48-55.

25. Bondemark L, Kurol J. Proximal alveolar bone level after orthodontic treatment with magnets, superelastic coils and straight-wire appliances. Angle Orthod. 1997; 67(1):7-14.

26. Demling A, Heuer W, Elter C, Heidenblut T, Bach FW, Schwestka-Polly R, StieschScholz M. Analysis of supra- and subgingival long-term biofilm formation on orthodontic bands.
Eur J Orthod. 2009; 31(2):2026.

27. Thornberg MJ, Riolo CS, Bayirli B, Riolo ML, Van Tubergen EA, Kulbersh R. Periodontal pathogen levels in adolescents before, during, and after fixed orthodontic appliance therapy. Am J Orthod Dentofacial Orthop. 2009; 135(1):95-8.

28. Choi DS, Cha BK, JostBrinkmann PG, Lee SY, Chang BS, Jang I, Song JS. Microbiologic changes in subgingival plaque after removal of fixed orthodontic appliances. Angle Orthod. 2009; 79(6):114955.

29. Sallum EJ, Nouer DF, Klein MI, Gonçalves RB, Machion L, Wilson Sallum A, Sallum EA. Clinical and microbiologic changes after removal of orthodontic appliances. Am J Orthod Dentofacial Orthop. 2004; 126(3):363-6.

30. Paolantonio M, di Girolamo G, Pedrazzoli V, di Murro C, Picciani C, Catamo G, Cattabriga M, Piccolomini R. Occurrence of A c t i n o b a c i l l u s actinomycetemcomitans in patients wearing orthodontic appliances. A cross-sectional study. J Clin Periodontol. 1996; 23(2):112-8.

31.Kessler M. Interrelationships between orthodontics and periodontics. Am J Orthod. 1976; 70(2):154-72.

32. Boyd RL, Leggott PJ, Quinn RS, Eakle WS, Chambers D. Periodontal implications of orthodontic treatment in adults with reduced or normal periodontal tissues versus those of adolescents. Am J Orthod Dentofacial Orthop. 1989; 96(3):191-8.

33. Artun J, Urbye KS. The effect of orthodontic treatment on periodontal bone support in patients with advanced loss of marginal periodontium. Am J Orthod Dentofacial Orthop. 1988; 93(2):143-8.

34. Nelson PA, Artun J. Alveolar bone loss of maxillary anterior teeth in adult orthodontic patients. Am J Orthod Dentofacial Orthop. 1997; 111(3):328-34.

35. Re S, Corrente G, Abundo R, Cardaropoli D. Orthodontic treatment in periodontally compromised patients: 12-year report. Int $\mathrm{J}$ Periodontics Restorative Dent. 2000; 20(1):31-9. 